\title{
ILT20 - an Upgraded Computer Program for Evaluation of Accelerated Leach Test Data of LLW in the Hungarian NPP Paks
}

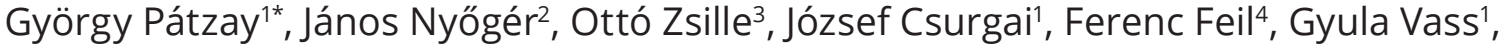 \\ Lajos Kátai Urbán', József Dobor ${ }^{1}$
}

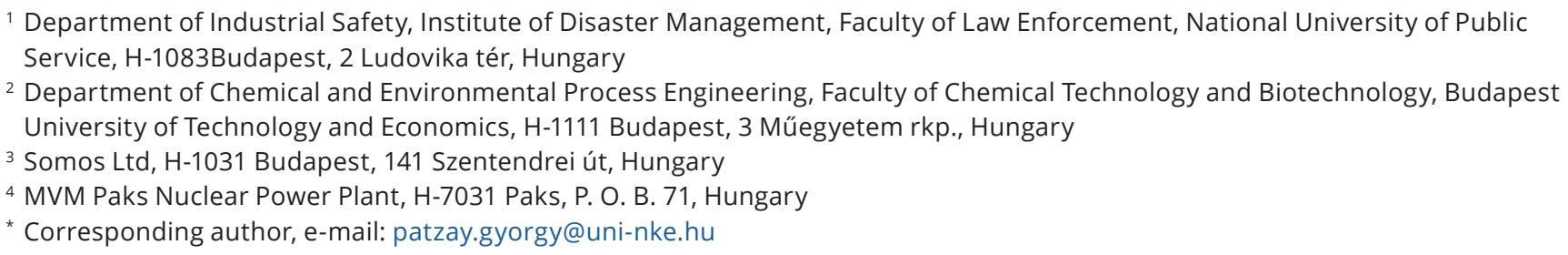

Received: 03 March 2021, Accepted: 09 June 2021, Published online: 16 August 2021

\begin{abstract}
Computer Program ILT15 was earlier developed to accompany a new leach test for solidified radioactive waste forms in the Hungarian NPP Paks. The program was designed to be used as a tool for performing the calculations necessary to analyze leach test data, a modelling program to determine if diffusion is the operating leaching mechanism (and, if not, to indicate other possible mechanisms), and a means to make extrapolations using the diffusion models. The program uses computational algorithm of ASTM C1308 standard. Now we have developed another computational algorithm based on a Partial Differential Equation (PDE) of a cylindrical specimen, solved the PDE by a Crank-Nicolson Finite Difference Method (FDM) and calculated by integration the eluted amount of a given component in time. The new solution more accurate method was integrated into the existing ILT15 computer program and the resulting new ILT20 program is able to calculate the Incremental/Cumulative Fraction Leached IFL/CFL) either by ASTM or PDE numerical solution methods.

ILT20 program is written in C++ in the Borland C++ Builder programming environment. A detailed description of application of this upgraded modelling computer program is given.
\end{abstract}

Keywords

accelerated leach test, computer program, Partial Differential Equation (PDE), Finite Difference Method (FDM), diffusion, fitting, cylinder

\section{Introduction}

Assessing the safety of radioactive waste disposal system is a complex process due to the dynamic nature of the hydrological and biological subsystems in the host environment that affects the degradation scenarios of the disposal facility. Safety assessment of the source term module is conducted by evaluating radionuclides release rates from the disposal facility as a function of time. This is achieved by studying degradation and/or failure mechanisms of the waste matrices, backfill, concrete barriers, cover system, and liner if exist. By the end of the containers lifetime, radionuclides releases are determined primarily by assessing the waste matrices performance. Cementation of radioactive waste has been practiced for many years basically for immobilization of low-and intermediate-level wastes to produce stable waste matrices with acceptable performance [1]. Studying radioactive isotope releases from cementitious waste matrices has received considerable attention; this is attributed to the low sorption potential of cement towards some of them (cesium) and solubility in the high $\mathrm{pH}$ environment of hydrated cement.

Researchers did an extended study to describe the leaching processes of radioactive or toxic materials from cement or other matrices. The aim of these studies was development of more accurate mathematical models and extrapolation of leaching for longer future times. Abdel Rahman and Zaki [2] directed an effort to assess the best source term conceptual model that represents Cs release 
from 14 different cementitious waste matrices. Cs releases from the studied matrices have been investigated according to IAEA recommended leach test. They used different source term conceptual models and their corresponding mathematical equations that represent the release from different cementitious waste matrices. The mathematical equations then used to estimate the leaching parameters. The experimental data were linearly and nonlinearly regressed to different developed source term conceptual models. The regression results indicated that Cs releases are best described by First-order Reaction/Diffusion Model (FRDM). FRDM regression results were compared to that of the IAEA recommended method to estimate the diffusion coefficients by performing statistical and uncertainty analysis.

Motny et al. [3] investigated the leaching behavior of cerium and cesium from different mixtures of Rapid Setting Cement (RSC) with deionized water (DIW) and artificial seawater (ASW) under both dynamic and static leach conditions according to the method in American National Standard ANSI/ANS-16.1-2003. The leachate solution was collected, diluted with $\mathrm{HNO}_{3}$, and analyzed using inductively coupled plasma-mass spectrometry after leaching periods of 2, 7, and $24 \mathrm{~h}$ and 2, 3, 4, 5, 14, 28, 43, and 90 days. Two models the First-order Reaction Model (FRM) and the Diffusion Model (DM) and their combination the First-order Reaction/Diffusion Model (FRDM) were fitted to assess the leaching parameters and identify the type of immobilized radionuclides in the RSC matrix. The result indicated that leaching of ${ }^{140} \mathrm{Ce}$ and ${ }^{133} \mathrm{Cs}$ from the RSC matrices with DIW and ASW under both dynamic and static leach conditions was less than $20 \%$ and suggested that the leaching behavior of ${ }^{140} \mathrm{Ce}$ and ${ }^{133} \mathrm{Cs}$ approximated that of a semi-infinite medium. The leaching phenomena of ${ }^{140} \mathrm{Ce}$ and ${ }^{133} \mathrm{Cs}$ could not be fully represented with a single model (the FRM and the DM), and the FRDM was best fitted to these experimental data sets. Overall, the average leachability index L values for ${ }^{140} \mathrm{Ce}$ and ${ }^{133} \mathrm{Cs}$ were greater than the minimum limit indicating their acceptance for disposal recommended by the International Atomic Energy Agency. It could also be stated that RSC with DIW or ASW had a potential for immobilizing radioactive materials.

Çoruh et al. [4] studied the leaching behavior of the zinc leach waste by utilizing a regression model with dummy variables. The results of different leaching methods indicate that addition of fly ash and blast furnace slag to the zinc leach waste reduces the heavy metal content in the effluent and that fly ash performs better than blast furnace slag. The metal release from the zinc leach waste decreased in relation to increasing treatment temperature.

Georget et al. [5] analysed a unified reactive transport model for leaching of cement paste in brines, with and without $\mathrm{CO}_{2}$. They observed distinct mechanisms as a function of the $\mathrm{pH}$ and the $\mathrm{CO}_{2}$ concentration of the leaching brine. In particular, clogging is observed at intermediate concentration of $\mathrm{CO}_{2}$ while higher concentration accelerates the reaction. In addition, they leveraged the flexibility of the simulator to explore the impact of different models on the simulations.

El-Kamash et al. [6] determined the transient mass release rates of radionuclides from an engineered disposal facility (cubic cement waste) due to waste matrix exhaustion. A two dimensional Partial Differential Equation (PDE) solved by a finite difference ADI method was used. The sensitivity analyses of certain input parameters to release rate calculation are also investigated. Results indicate that the disposal facility with clay and crushed rock backfill materials could contain almost all the radionuclides normally encountered in low level radioactive waste, where short-lived radionuclides having higher distribution coefficient values are completely confined within the waste form. On the other hand, inventories of long lived radionuclides having lower distribution coefficient values need to be regulated so as to reduce their entry into the geosphere.

For a number of years increasing attention has been given in Hungary to the management of the low and medium level radioactive wastes (LLW, MLW) being produced in Paks nuclear power plant.

Some of these wastes, for example, evaporator bottom concentrates, pond sludge and spent ion exchange media are produced in relatively large volumes. In addition to national programs on the development of immobilization processes, the European Community commissioned programs on the immobilization of LLW and MLW. These wastes are immobilized by incorporating them into cement. In order to optimize these immobilization processes, for example with respect to waste loading, it was necessary to characterize the products with respect to such properties as density, strength, dimensional stability, leach resistance and so on.

Computer Program ILT15 was developed in 2015 to accompany a new leach test for solidified radioactive waste forms. The program was designed to be used as a tool for performing the calculations necessary to analyze 
leach test data, a modelling program to determine if diffusion is the operating leaching mechanism (and, if not, to indicate other possible mechanisms), and a means to make extrapolations using the Diffusion Models. The ILT15 program contains four mathematical models that can be used to represent the data [7-10].

This work is an extension for our previous works, which were conducted using ASTM C1308-08 (2017) standard for accelerated leach test experiments and constructed a computer program ILT15 [11]. The extension is based on a new, more accurate numerical solution of diffusion mass transfer Partial Differential Equations in cylindrical specimen. the upgraded new computer program (ILT20) is written in $\mathrm{C}++$ in the Borland $\mathrm{C}++$ Builder programming environment. ILT20 was designed to be used as a tool for performing the calculations necessary to analyze leach test data, a modelling program to determine if diffusion is the operating leaching mechanism (and, if not, to indicate other possible mechanisms), and a means to make extrapolations using the Diffusion Models.

The mathematical models describing leaching mechanisms are as below:

1. Diffusion through a semi-infinite medium (for low fractional releases),

2. Diffusion through a finite cylinder (for high fractional releases),

3. Diffusion plus partitioning of the source term,

4. Solubility limited leaching.

The program uses simple mathematical models described in the ASTM C1308-08 (2017) standard [11].

\section{Methododolgy for leaching by diffusion}

Mass transfer via diffusion is described by the Fick laws. In simplest case the diffusion is not depends on time and described by the Fick ${ }^{1 \text { st }}$ law:

$$
\begin{gathered}
\Phi_{m}=-D \frac{\Delta c}{\Delta x} \\
\Phi_{m}=\frac{\Delta m}{\Delta t \times A}
\end{gathered}
$$

where:

$\Phi_{m}$ mass flow density (mass flux), mass of $\mathrm{kg} /\left(\mathrm{m}^{2} \mathrm{~s}\right)$ material diffused through a unit of area during unit of time

$D$ diffusion coefficient $\mathrm{m}^{2} / \mathrm{s}$

$C$ mass concentration $x \quad$ diffusion path parallel with

$\mathrm{m}$

$m$ mass in diffusion

$A$ area (cross section) normal to diffusion $\mathrm{m}^{2}$

$t$ time $\mathrm{kg}$

This process is ideal, supposing timeless inflow of diffusing material and constant concentrations in time at various distances.

In case of changing concentrations in space and time the Fick $2^{\text {nd }}$ law describes the phenomena:

$\frac{\Delta C}{\Delta t}=D \frac{\Delta\left(\frac{\Delta C}{\Delta x}\right)}{\Delta x}$

where:

$\frac{\Delta\left(\frac{\Delta C}{\Delta x}\right)}{\Delta x}$ is the linear concentration gradient change in space.

In general form:

$\Phi_{m}=-D \frac{\Delta c}{\Delta x} \quad$ Fick $1^{\text {st }}$ law

$\frac{\partial c}{\partial t}=-D \nabla^{2} c \quad$ Fick $2^{\text {nd }}$ law.

\subsection{Basics of the test and requirements for the test components}

Base of leach test is a semi dynamic method, when a cylindrical specimen is immersed in a leach solution (water or aqueous solution), then usually in time the specimen is exchanged with a new one end the leached concentration or mass is determined. This compared to the original total concentration or mass results the Incremental Fraction Leached (IFL). Summing the IFL values till a given leach time we get the Cumulative Fraction Leached (CFL) values. More frequent exchange of specimens during the test results more exact modelling with the Fick $2^{\text {nd }}$ law, but the leached amount of material will be lower, and the determination uncertainty will be larger. Because of the above restrictions the leaching time intervals are optimized. For that reason, the leach test should be completed under standard conditions, including the specimen and leach solution characteristics as well as the leach vessel material and auxiliary conditions (specimen fixation, mixing, filtering etc.):

1. Requirements for the leaching liquor:

- leach solution will not react with the material of the specimen and will not modify it

- leach solution should not contain such a component, which modifies the leaching mechanism. 
2. Requirements for the leaching vessel:

- wall of the vessel could not react with the solution and leached components

- exchange of the solution should be easy and the solution in the vessel should not evaporate.

3. Requirements for the auxiliary components:

- their materials should not react with the solvent and with the leached materials

- filtered leached materials could be analyzed

- filter will remove particles with diameter $>45 \mu \mathrm{m}$

- hanging the specimen should not influence the leaching and should not cover more than $1 \%$ of the surface.

4. Requirements for the specimen:

- specimen is cylindrical body with a diameter/ height ratio $1 / 1$ and their value is $2.5 \mathrm{~cm}$

- specimen composition should be identical with the waste composition

- distribution of the radioactive isotope(s) or heavy metal material should be uniform in specimen

- the structure of the specimen material should be the identical at the surface and in the bulk

- every specimen geometry, mass and embedded radioactive or heavy metal content should be accurately determined.

5. Other requirements:

- the temperature during leaching should be constant with a maximum fluctuation less then: $1{ }^{\circ} \mathrm{C}$

- surface to volume ratio for specimen should be constant during leach test(s) and the ratio should be:

$$
S_{V}=\frac{S_{S}}{V_{L}}=0.1(1 / \mathrm{cm})
$$

where:

$\begin{array}{lll}S_{V} & \text { specific surface } & 1 / \mathrm{cm} \\ S_{S} & \text { specimen surface } & \mathrm{cm}^{2} \\ V_{L} & \text { volume of leaching liquid } & \mathrm{cm}^{3}\end{array}$

- Regularly at determined term in the leaching liquid should be changed the amount of the leached material or activity should be determined. These intervals should be from the start of leaching $2 \mathrm{~h}, 7 \mathrm{~h}, 24 \mathrm{~h}, 48 \mathrm{~h}$, till the end of the $11^{\text {th }}$ day.

Using the determined leached amounts of material(s) or activities one can calculate the Incremental/Cumulative Fraction Leached IFL/CFL):

$$
\begin{aligned}
\mathrm{IFL} & =\frac{a_{i, j}}{A_{i, 0}} \\
\mathrm{CFL} & =\frac{\sum_{j=1}^{n} a_{i, j}}{A_{i, 0}}=\sum_{j=1}^{n} \mathrm{IFL}_{j}
\end{aligned}
$$

where:

$i \quad$ index of the radioactive isotope or heavy metal

$j \quad$ index of the leach time interval

$a_{i, j} \quad$ leached in the actual time interval activity $\mathrm{Bq}$ (concentration) for the actual isotope or heavy metal

$A_{i, 0} \quad$ activity or concentration of the $i$-th radioactive $\mathrm{Bq}$ isotope or heavy metal in the specimen before the start of leaching

Using the IFL/CFL values the $D_{e}$ effective diffusion coefficient could be determined. Accuracy of fitting of the leach data could be characterized by Eq. (7):

$E_{R^{2}}=\frac{\sum_{j=1}^{n}\left(\mathrm{CFL}_{j, \text { model }}-\mathrm{CFL}_{j, \text { measured }}\right)^{2}}{\mathrm{CFL}_{n \text {, measured }}}$

where:

$$
\begin{array}{ll}
E_{R^{2}} & \begin{array}{l}
\text { calculated CFL values at a given fitting } \\
\text { model }
\end{array} \\
n & \text { number of leaching time intervals } \\
\mathrm{CFL}_{j, \text { model }} & \begin{array}{l}
\text { calculated by fitting model CFL value } \\
\text { at the } j \text {-th interval }
\end{array} \\
\mathrm{CFL}_{j, \text { measured }} & \begin{array}{l}
\text { measured leached CFL value at the } j \text {-th } \\
\text { interval }
\end{array} \\
\mathrm{CFL}_{n, \text { measured }} & \text { measured leached CFL value at the } n \text {-th } \\
& \text { interval (sum) }
\end{array}
$$

A fitting model is suitable to describe the measured leaching CFL data if $E_{R^{2}}<0.5 \%$. The lower the $E_{R^{2}}$ the higher is the accuracy of the fitting algorithm.

\subsection{Leaching model used in the ILT20 program}

\subsubsection{Diffusion leaching model}

Radial diffusion in a solid cylinder could be described by a second order linear parabolic Partial Differential Equation:

$$
\frac{\partial c(r, t)}{\partial t}=D \times \frac{\partial^{2} c(r, t)}{\partial r^{2}}+D \times \frac{1}{r} \times \frac{\partial c(r, t)}{\partial r}
$$

where $c\left[\mathrm{~mol} / \mathrm{dm}^{3}, \mathrm{~g} / \mathrm{dm}^{3}, \ldots\right.$ etc.] concentration, $r$ [cm] radial distance from the axis of cylinder, $t[\mathrm{~s}]$ time and 
$D\left[\mathrm{~cm}^{2} / \mathrm{s}\right]$ effective diffusion coefficient. Equation (8) could be transformed into dimensionless form by using the following variables:

- dimensionless concentration: $u[-]=C / C_{0}$, where $C_{0}$ is the initial concentration;

- dimensionless radius: $\rho[-]=r / R$, where $R$ is the radius of cylinder;

- dimensionless time: $\tau[-]=t \times D / R^{2}$.

Using dimensionless variables, we get

$\frac{\partial u(\rho, \tau)}{\partial \tau}=\frac{\partial^{2} u(\rho, \tau)}{\partial \rho^{2}}+\frac{1}{\rho} \times \frac{\partial u(\rho, \tau)}{\partial \rho}$

where $u$ and $\rho$ values are between 0 and 1 .

At $t=0(\tau=0)$ the concentration in cylinder is everywhere equal to $C_{0}$ so:

$u(\rho, 0)=1 \quad(\forall 0 \leq \rho \leq 1)$.

The boundary conditions are:

$\partial u / \partial \rho(0, \tau)=0 \quad(\forall \tau)$

$u(1, \tau)=0 \quad(\forall \tau>0)$.

The parabolic Partial Differential Equation could be solved numerically by a finite difference scheme.

Using a finite difference solution, the dimensionless radius between [0;1] was divided into $N$ equal length step, so the uniform step size is $\Delta \rho=1 / N$, and the value of $\rho$ and $u_{i}$ could be calculated by

$\rho_{i}=i \times \Delta \rho$

where $i=0,1,2, \ldots, N$.

While the value of dimensionless radius could change between 0 and 1 , the value of dimensionless time starts from 0 and could increase without limit. The dimensionless time ant $j$-th time step:

$\tau_{j}=j \times \Delta \tau$

where $j=0,1,2, \ldots, T$, and $T$ is the number of time steps. So, the dimensionless concentration in the cylinder at a given $i$-th point of radius and $j$-th point of time:

$u_{i, j}=u\left(\rho_{i}, \tau_{j}\right)$

For the finite difference approximation of the Eq. (8) by Crank-Nicholson method many cases it is better to use points shifted from the boundaries by $1 / 2 \times \Delta \rho$ [12]. In this case Eq. (13) changes as (see Fig. 1):

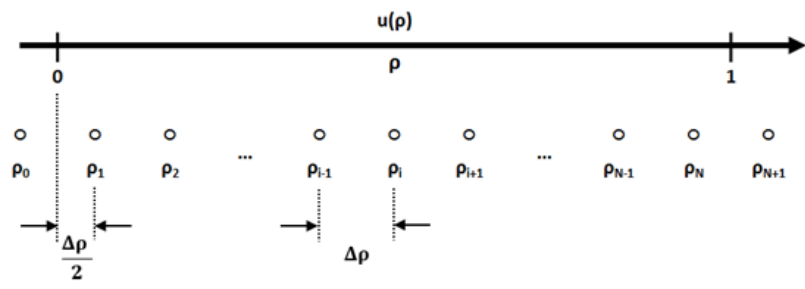

Fig. 1 Points shifted from the boundary

$\rho_{i}=(i-1 / 2) \times \Delta \rho$

and $i=1,2,3, \ldots, N . i=1$ and $i=N$.

Using these discretizations Eq. (8) could be described for the first space step $(i=1)$, intermediate points $(2 \leq i \leq N-1$ and for the last point $(i=N)$ using the CrankNicholson scheme and the boundary and initial conditions after rearrangement as follows:

$\left(1+\frac{(\Delta \rho)^{2}}{\Delta \tau}\right) u_{1, j+1}-u_{2, j+1}=\left(-1+\frac{(\Delta \rho)^{2}}{\Delta \tau}\right) u_{1, j+1}-u_{2, j+1}$,

$\left(-\frac{i-1}{2 i-1}\right) u_{i-1, j+1}+\left(1+\frac{(\Delta \rho)^{2}}{\Delta \tau}\right) u_{i, j+1}+\left(-\frac{i}{2 i-1}\right) u_{i+1, j+1}$

$=\left(\frac{i-1}{2 i-1}\right) u_{i-1, j}+\left(-1+\frac{(\Delta \rho)^{2}}{\Delta \tau}\right) u_{i, j}+\left(\frac{i}{2 i-1}\right) u_{i+1, j}$,

$\left(-\frac{N-1}{2 N-1}\right) u_{N-1, j+1}+\left(1+\frac{(\Delta \rho)^{2}}{\Delta \tau}+\frac{N}{2 N-1}\right) u_{N, j+1}$

$=\left(\frac{N-1}{2 N-1}\right) u_{N-1, j}+\left(-1+\frac{(\Delta \rho)^{2}}{\Delta \tau}-\frac{N}{2 N-1}\right) u_{N, j}$.

The resulting set of algebraic equations forms a tridiagonal matrix (Eq. (20)) and could be solved by the Thomas algorithm.

$b_{1} \times u_{1}+c_{1} \times u_{2}+0+\ldots+0=d_{1}$

$a_{2} \times u_{1}+b_{2} \times u_{2}+c_{2} \times u_{3}+0+\ldots+0=d_{2}$

...

$0+\ldots+0+a_{i} \times u_{i-1}+b_{i} \times u_{i}+c_{i} \times u_{i+1}+0+\ldots+0=d_{i}$

$0+\ldots+0+a_{N-1} \times u_{N-2}+b_{N-1} \times u_{N-1}+c_{N-1} \times u_{N}=d_{N-1}$

$0+\ldots+0+a_{N} \times u_{N-1}+b_{N} \times u_{N}=d_{N}$.

Using the above calculations, we can get the dimensionless concentrations in the cylindrical specimen along the radius at any time. Fig. 2 shows an example of calculated radial concentrations at the selected points as a function of time.

The Crank-Nicholson method of FDM is stable if:

$\frac{\Delta \tau}{(\Delta \rho)^{2}} \leq 0.5$. 


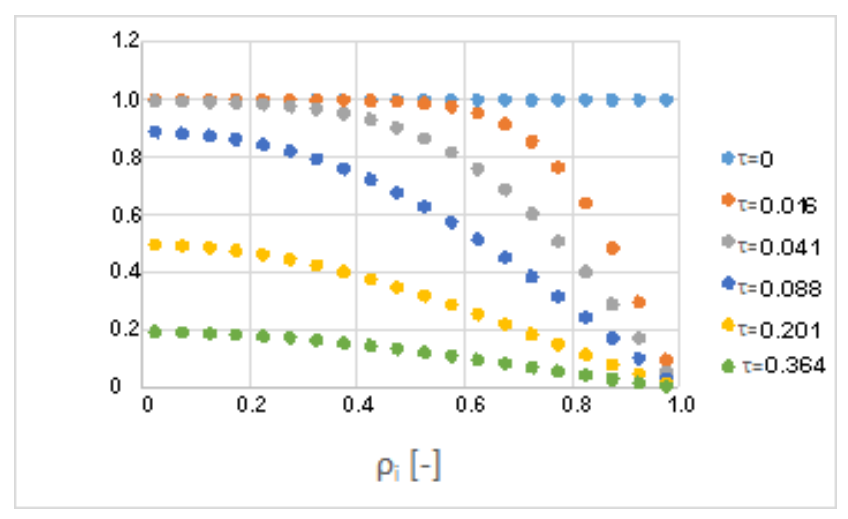

Fig. 2 Concentration profile along the radius at different time $(N=20)$

\subsubsection{Calculation of CFL values}

The calculated radial concentration profile at a given time is uniform in any direction and describes a symmetrical body, which integrated volume determines the remaining concentration in the cylindrical specimen. The total remaining amount of radioactivity (or toxic metal) so could be determined. Subtracting the remaining amount at a given leach time from the beginning saturation amount we get the leached amount and the CFL value. At $\tau=0$, the volume is a cylinder, with a dimensionless volume $1^{2} \times \pi \times 1=\pi$. At $\tau_{j}>0$ the remaining the integrated volume is $V_{j}$, and the CFL could be determined:

$$
\mathrm{CFL}_{j}=\frac{\pi-V_{j}}{\pi} \text {. }
$$

$V_{j}$ could be determined as follows. First the area below the concentration profile $\left(u_{i}-\rho_{i}\right)$ was approximated by the trapezoid rule (Eq. (23)), then the first coordinate of the centre of gravity of the given polygon was determined (Eq. (24)). The volume was then determined by the Pappus-Guldin theorem 2 (Eq. (25)).

$$
\begin{aligned}
& A \approx \Delta \rho \times\left(\sum_{i=1}^{N} u_{i}-0.25 \times u_{N}\right) \\
& C_{x}=\frac{1}{6 A} \times\left[\begin{array}{l}
0.5 \times \Delta \rho \times\left(-0.5 \times \Delta \rho \times u_{1}\right) \\
+\sum_{i=1}^{N-1}((i-1 / 2) \times \Delta \rho+(i+1 / 2) \times \Delta \rho) \\
\times\left((i-1 / 2) \times \Delta \rho \times u_{i+1}-(i+1 / 2) \times \Delta \rho \times u_{i}\right) \\
+((N-0.5) \times \Delta \rho+1) \times\left(-u_{N}\right)
\end{array}\right]
\end{aligned}
$$

$V_{j}=A_{j} \times C_{x, j} \times 2 \pi$

Fig. 3 shows a CFL-leach time curves with different effective diffusion coefficients.

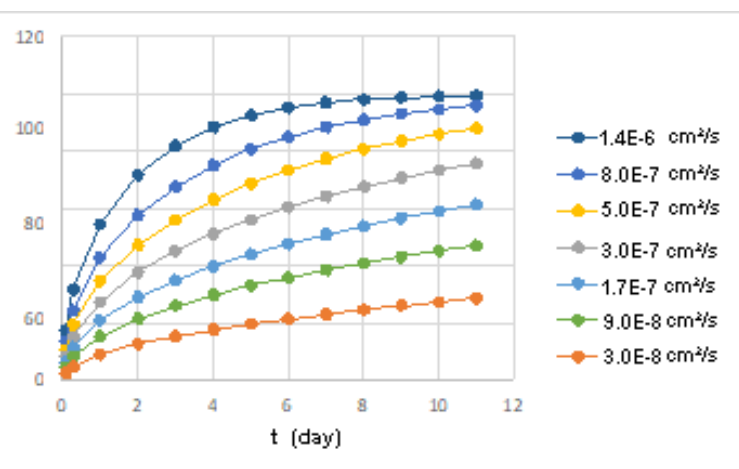

Fig. 3 Calculated CFL values as a function of time at various diffusivities

\subsubsection{Fitting of the calculated CFL values on the measured CFL values}

Best fit on the measured leach values (CFL) is obtained by optimal change of the parameters till the percent relative error is less than $0.5 \%$. The changed parameter in case of pure diffusional leach is the effective diffusion coefficient, in case of mixed leach by diffusion and partition are the effective diffusion coefficient and partitioning factor both. The percent relative error in the fit of the model to the data is determined by dividing the sum of the squares of the residuals between the CFL value of the fitted model curve and the measured value by the CFL value of the experimental data of the longest duration.

$E_{R^{2}}=100 \times \frac{\sum_{j=1}^{N^{\prime}}\left(\mathrm{CFL}_{j, \text { model }}-\mathrm{CFL}_{j, \text { meas }}\right)^{2}}{\mathrm{CFL}_{N^{\prime}, \text { meas }}}$

The computation process is based on iteration till the percent relative error decreases.

\section{Validation of the ILT20 program}

\subsection{Running the program and comparing programs ILT15 and ILT20}

When the program begins to run after installation the following main menu will appear on the screen waiting for the input data from the keyboard or from a "csv" data file.

We can enter from the keyboard the measured leach data from experimental results (e.g., leach time and counts per minute-cpm, or -concentration). Additionally, we inputted the height and diameter of the solid cylindrical specimen, volume and surface and material of leachant, and the number of summa count of radioactivity or concentration. Alternatively, we can input leaching data from an earlier saved "csv" data file. 
As an example, we inputted Cs-137 leach test data using the leach test data measured from a c400 cement cylinder with the embedded evaporator bottom residue of the tank 02TW10B002 of the NPP (Fig. 4).

After completing data input (and/or editing) we selected from the "Calculation" menu the fitting model form the "Calculation" menu. During calculations we took account of the possible decays during leach time. We selected first the "Leach by diffusion plus partitioning" according to ILT15 (ASTM C1308-08 (2017) standard). The result of model fit is seen in Figs. 5 and 6.

It is seen from fitting results, that the selected leach model and calculation method describes the measured values quite well, with $D_{e}=4.809 \times 10^{-7} \mathrm{~cm}^{2} / \mathrm{s}$ and partition coefficient $C=0.817, E_{R^{2}}=1.547 \%$, which is higher then $0.5 \%$.

Choosing the PDE-FDM model with diffusion plus partitioning using "Calculation" and model 4 and switching out the "Only diffusion" checkbox the results of fitting are shown in Figs. 7 and 8.

It is seen from fitting results, that the selected leach model and calculation method describes the measured values very well, with $D_{e}=2.24 \times 10^{-6} \mathrm{~cm}^{2} / \mathrm{s}$ and partition coefficient $C=0.730, E_{R^{2}}=0.065 \%$. So the new PDE-FDM modelling is more accurate, because its $E_{R^{2}}$ is lower (0.065\%).

The new ILT20 program is also able to extrapolate the calculated CFL data for longer leaching time also, for maximum 1000 days. For example, using in "Calculation" model 2

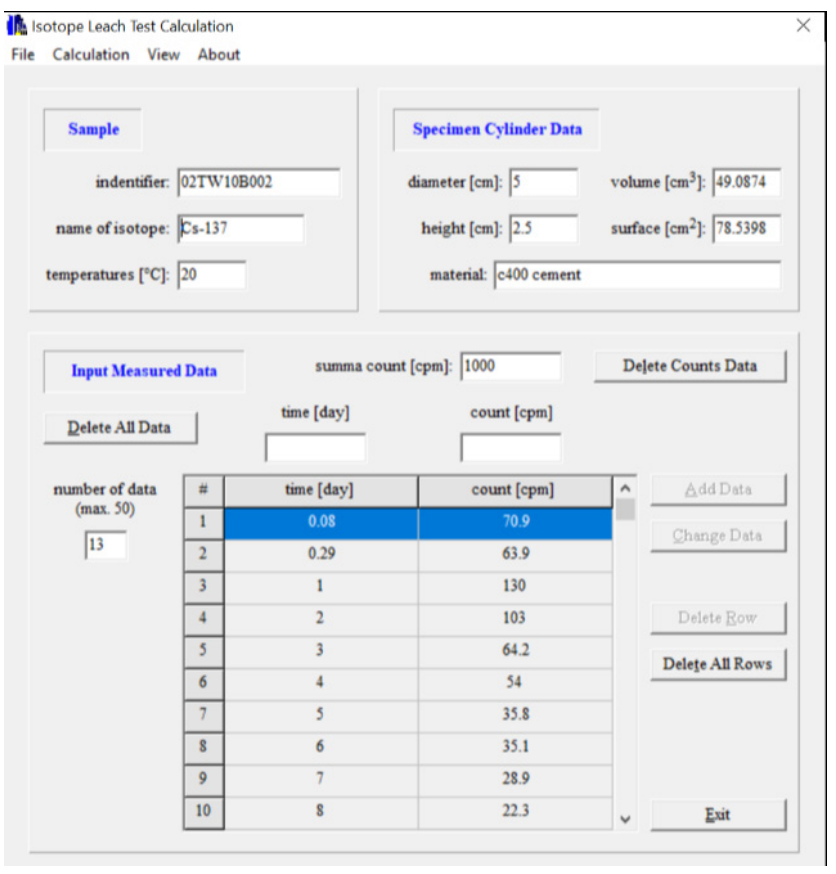

Fig. 4 Leach data measured from a c400 cement cylinder with the embedded evaporator bottom residue of the tank 02TW10B002 of the NPP

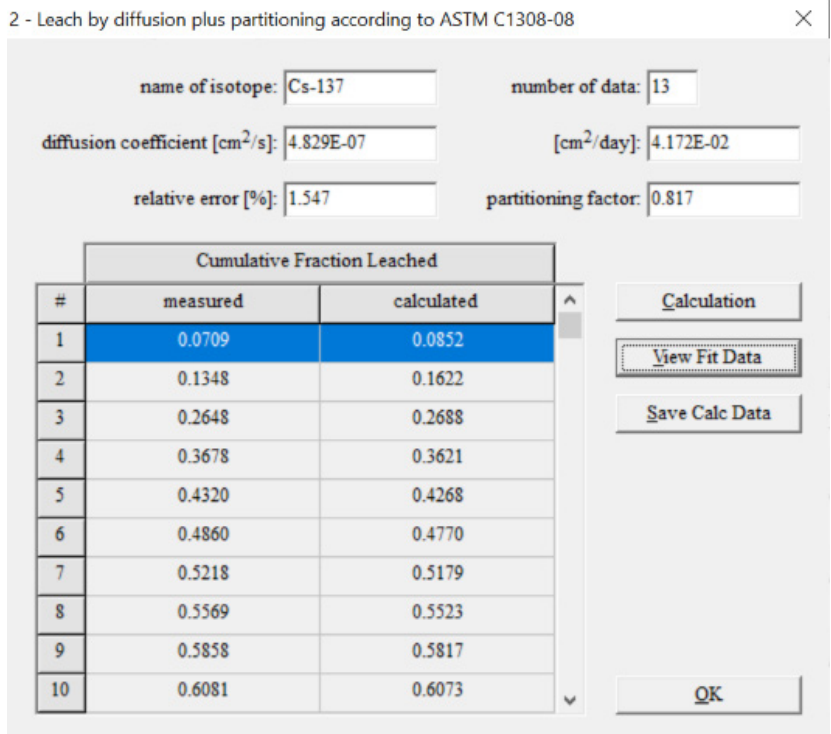

Fig. 5 Fitting results of "Leach by diffusion plus partitioning according to ASTM C1308-08" standard

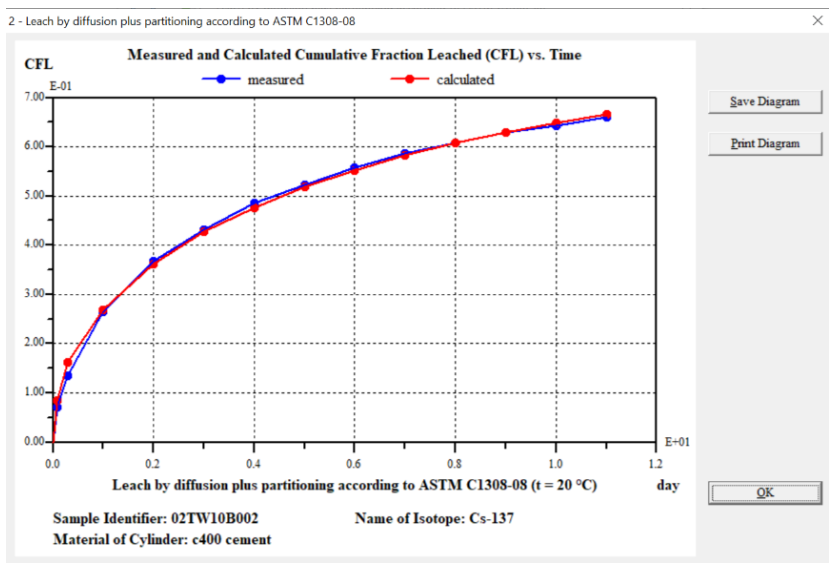

Fig. 6 Fitting results of "Leach by diffusion plus partitioning according to ASTM C1308-08" standard

and selecting the "Extrapolation" submenu the extrapolated leaching data for 200 days are seen in Figs. 9 and 10.

\section{Results}

The developed new ILT20 computer program was tested by comparing the modelling results determined by the PDF-FDM and by the ASTM C1308-08 (2017) standard calculation methods for diffusion plus partitioning modell. Comparison was made with the accelerated leach test data of important radioactive isotopes of NPP waste.

Results are shown in Table 1.

Based on these modelling results we concluded, that the new ILT20-FDM method is more accurate, than ILT15 and this is due the more accurate description of the leach curve at small CFL values (see Fig. 11) 


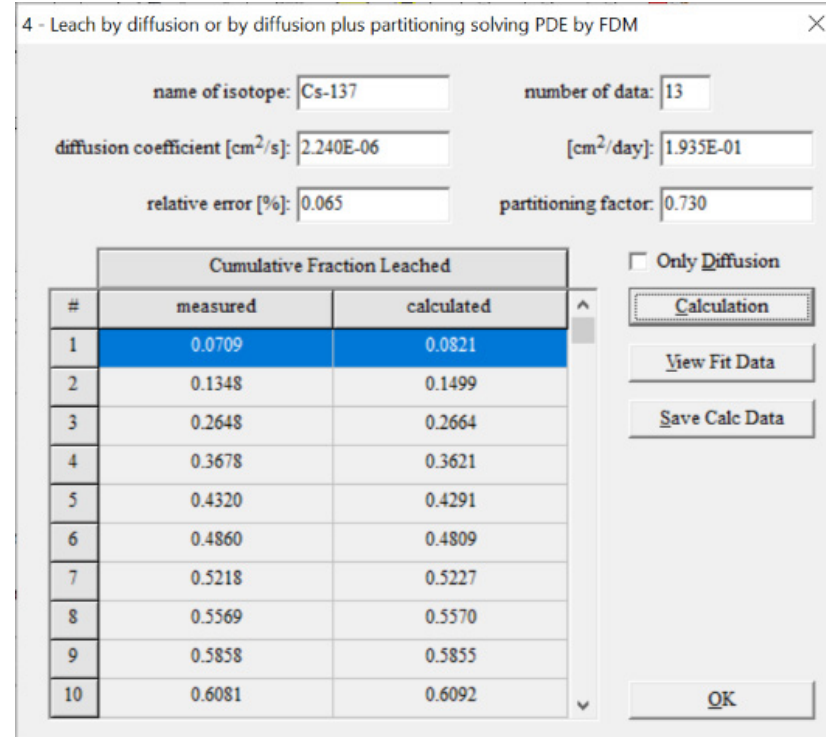

Fig. 7 Fitting results of "Leach by diffusion plus partitioning according to PDE by FDM"

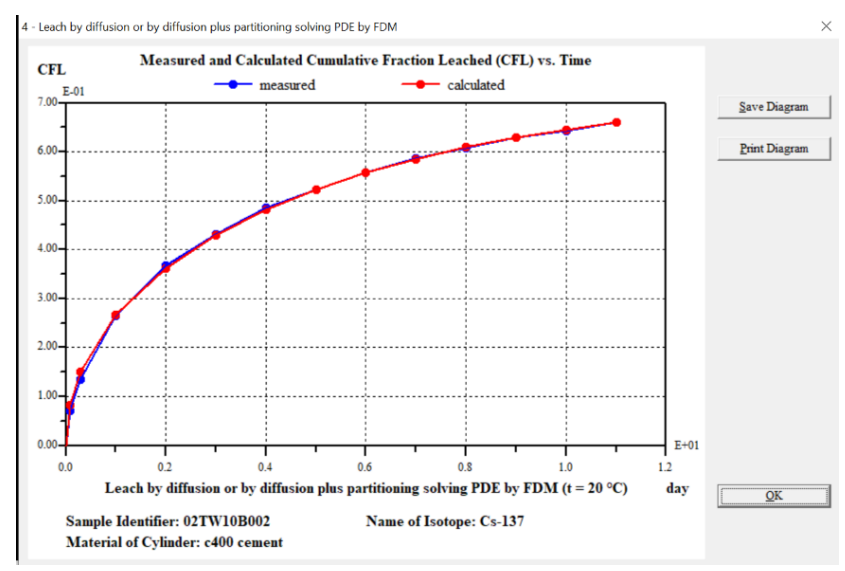

Fig. 8 Fitting results of "Leach by diffusion plus partitioning according to PDE by FDM"

\section{Conclusion}

The upgraded new ILT20 computer modelling program is able to fit measured leach data (CFL-time) with four different simulation algorithms:

1. Leach by diffusion according to ASTM C1308-08 (2017),

2. Leach by diffusion plus partitioning according to ASTM C1308-08 (2017),

3. Solubility limited leaching according to ASTM C1308-08 (2017),

4. Leach by diffusion or diffusion plus partitioning solving PDE by FDM method.

Additionally, it is able to extrapolate the modelled CFL values for longer leaching time (till 1000 days). According

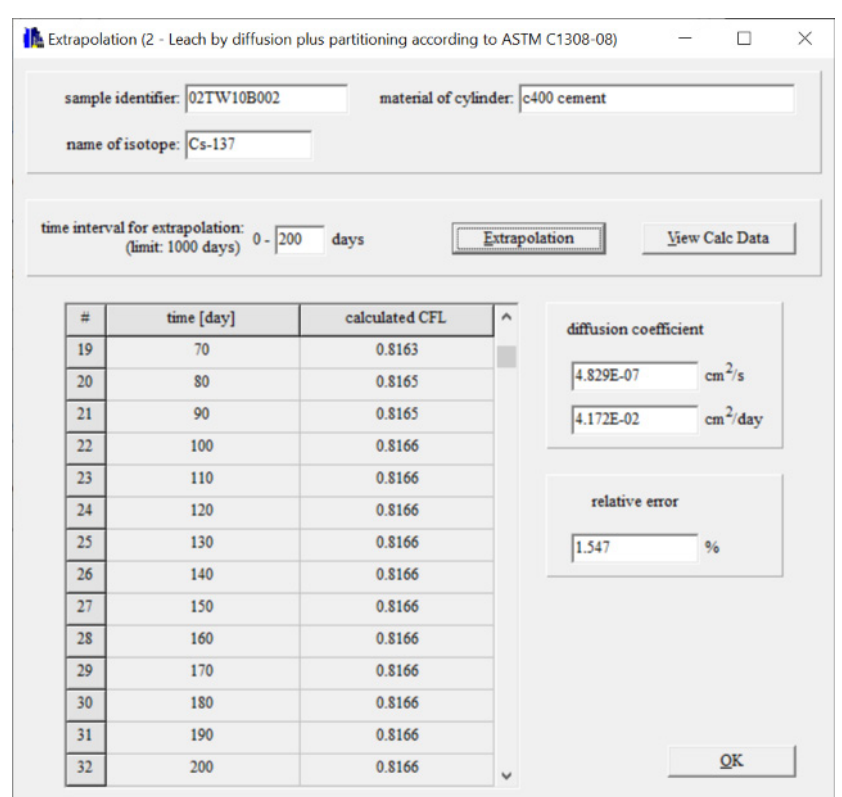

Fig. 9 Extrapolated CFL values till 200 days

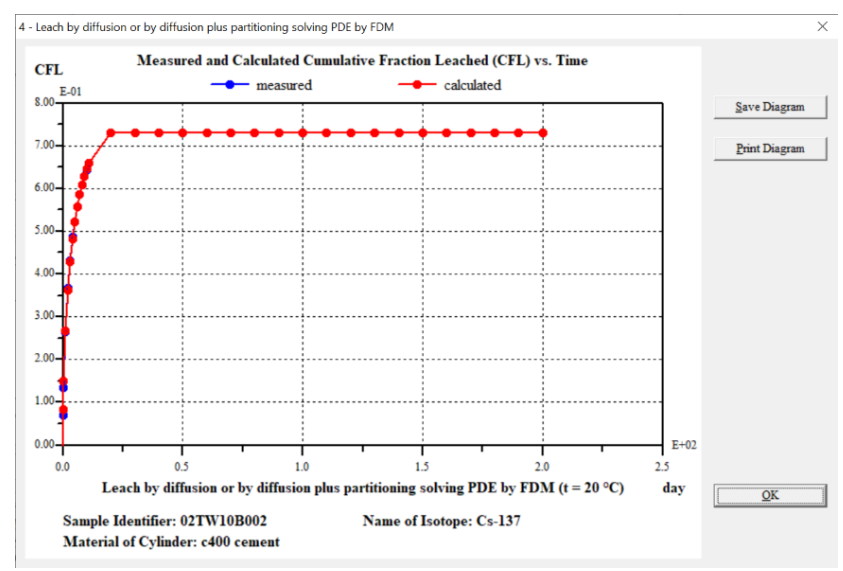

Fig. 10 Extrapolated CFL values till 200 days

to results the new incorporated modelling and calculation method, namely the parabolic Partial Differential Equation (PDE) for the cylindrical test specimen solved by a Finite Difference Method (FDM), using the CrankNicholson method, result more accurate fit to measured leach test data in case of pure diffusion as well as diffusion plus portioning. This development increased the accuracy of the model fitted on the measured data, allowing more accurate extrapolation for longer leach time.

To test the developed new ILT20 program we used the measured data of leaching Ag-110m, Ba-133, Co-60, Cs-137, Mn-54 from c400 cement matrix in case of embedded evaporator bottom residue (tank 02TW10B002) from the Paks NPP. 
Table 1 Fitting results of ILT20 and ILT15

\begin{tabular}{llcc}
\hline & & \multicolumn{2}{c}{ Diffusion plus partitioning } \\
& & ILT20-FDM & ILT15 \\
\hline \multirow{3}{*}{$110 \mathrm{~m}_{\mathrm{Ag}}$} & $D e\left[10^{-7} \mathrm{~cm}^{2} / \mathrm{s}\right]$ & 6.417 & 3.124 \\
& $P[-]$ & 0.7467 & 0.7866 \\
& $E_{R^{2}}[\%]$ & 0.1035 & 0.1226 \\
\hline \multirow{3}{*}{$133_{\mathrm{Ba}}$} & $D e\left[10^{-7} \mathrm{~cm}^{2} / \mathrm{s}\right]$ & 6.199 & 2.974 \\
& $P[-]$ & 0.7413 & 0.7842 \\
& $E_{R^{2}}[\%]$ & 0.0756 & 0.1656 \\
$60_{\mathrm{Co}}$ & $D e\left[10^{-7} \mathrm{~cm}^{2} / \mathrm{s}\right]$ & 6.012 & 2.840 \\
& $P[-]$ & 0.7400 & 0.7864 \\
& $E_{R^{2}}[\%]$ & 0.0804 & 0.1456 \\
\hline \multirow{3}{*}{$137_{\mathrm{Cs}}$} & $D e\left[10^{-7} \mathrm{~cm}^{2} / \mathrm{s}\right]$ & 5.600 & 2.567 \\
& $P[-]$ & 0.7298 & 0.7827 \\
& $E_{R^{2}}[\%]$ & 0.0655 & 0.1212 \\
\hline \multirow{3}{*}{$54_{\mathrm{Mn}}$} & $D e\left[10^{-7} \mathrm{~cm}^{2} / \mathrm{s}\right]$ & 5.554 & 2.536 \\
& $P[-]$ & 0.7253 & 0.7786 \\
& $E_{R^{2}}[\%]$ & 0.0259 & 0.2040 \\
\hline
\end{tabular}

\section{References}

[1] Abdel Rahman, R. O., Zaki, A. A. "Comparative study of leaching conceptual models: Cs leaching from different ILW cement based matrices", Chemical Engineering Journal, 173(3), pp. 722-736, 2011. https://doi.org/10.1016/j.cej.2011.08.038

[2] Abdel Rahman, R. O., Zaki, A. A. "Comparative analysis of nuclear waste solidification performance models: Spent ion exchanger-cement based wasteforms", Process Safety andEnvironmental Protection, 136, pp. 115-125, 2020.

https://doi.org/10.1016/j.psep.2019.12.038

[3] Motny, R. M., Woods, M. E. Phongikaroon, S. "Assessment of Leaching Characteristics for Cerium and Cesium as Surrogates for Radioactive Materials", Nuclear Technology, 206(12), pp. 1932-1944, 2020.

https://doi.org/10.1080/00295450.2020.1730672

[4] Çoruh, S., Elevli, S., Ergun, O. N., Demir, G. "Assessment of leaching characteristics of heavy metals from industrial leach waste", International Journal of Mineral Processing 123 pp. 165-171, 2013. https://doi.org/10.1016/j.minpro.2013.06.005

[5] Georget, F., Prévost, J. H., Huet, B. "Reactive transport modelling of cement paste leaching in brines", Cement and Concrete Research, 111, pp. 183-196, 2018.

https://doi.org/10.1016/j.cemconres.2018.05.015

[6] El-Kamash, A. M., Mohamed, R. O., Nagy, M. E., Khalill, M. Y. "Modeling and validation of radionuclides releases from an engineered disposal facility", International Journal of Waste Management and Environmental Restoration, 22(4), pp. 373-393, 2002.

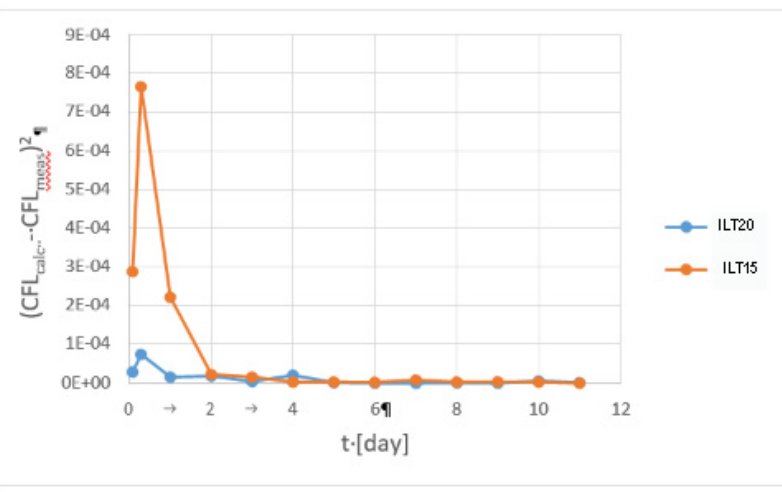

Fig. 11 Square of deviations for ILT15 and ILT20 as a function of leaching time for Mn-54

[7] Pátzay, G., Zsille, O., Csurgai, J., Nényei Á., Feil, F., Vass, G. "ILT15 - A Computer Program for Evaluation of Accelerated Leach Test Data of LLW in the Hungarian NPP Paks", Periodica Polytechnica Chemical Engineering, 63(3), pp. 527-532, 2019. https://doi.org/10.3311/PPch.11714

[8] Machiels, A. J., Pescatore, C. "Modeling of Waste Form Leaching, Part I Status of Leach Modeling", University of Illinois, Champaign, IL, USA, Rep. UILU-ENG-82-5319, 1982.

[9] Pescatore, C. "Improved expressions for modeling diffusive, fractional cumulative leaching from finite-size waste forms", Waste Management, 10(2), pp. 155-159, 1990.

https://doi.org/10.1016/0956-053X(90)90120-A

[10] United States Environmental Protection Agency (EPA) "SW-846 Test Method 1315: Mass Transfer Rates of Constituents in Monolithic or Compacted Granular Materials Using as Semi-Dynamic Tank Leaching Procedure", Washington, D.C., USA, 2017.

[11] ASTM International "ASTM C1308-08 (2017) Standard Test Method for Accelerated Leach Test for Diffusive Releases from Solidified Waste and a Computer Program to Model Diffusive, Fractional Leaching from Cylindrical Waste Forms", ASTM International, West Conshohocken, PA, USA, 2017. https://doi.org/10.1520/C1308-08R17

[12] Von Rosenberg, D. U. "Methods for the numerical solution of partial differential equations", American Elsevier Publishing Company, Inc, New York, NY, USA, 1969. 